\title{
AN ETHNOBOTANICAL EXPLORATION OF MEDICINAL PLANTS IN MANAR BEAT, KARAMADAI RANGE, WESTERN GHATS, TAMIL NADU
}

\author{
RAMYA EK*, MOWNIKA S, SHARMILA S
}

Department of Botany, Vellalar College for Women (Autonomous), Thindal, Erode, Tamil Nadu, India. Email: ekramya16@gmail.com

Received: 12 June 2019, Revised and Accepted: 15 July 2019

\section{ABSTRACT}

Objectives: This investigation provides an ethnobotanical information that truly focused on the traditional medicinal plants used by Irula community inhaled in Manar beat to treat various human diseases. Ethnobotanical study about medicinal plants was carried out from January 2018 to December 2018 in Manar beat, Karamadai range, Western Ghats, Tamil Nadu.

Methods: The traditional in-depth knowledge of medicinal plants was collected during group discussion, interviews, and guided field walks along with tribe. All the traditional medicinal plants collected during the field visit were identified with local floras and the identity was authenticated by Botanical Survey of India.

Results: A total of 89 medicinally important endemic, threatened and endangered aromatic herbal plants distributed in 71 genera and 42 families were collected and identified. Among the surveyed plants, Fabaceae and Moraceae with eight species were the largest plant families, respectively. Leaves (35\%) are most widely used plant part of reported plants and decoction forms are mostly used by Irulas. The surveyed plants were checked for conservation status in Red Data List formulated by the International Union for Conservation of Nature; around nine species were listed out.

Conclusion: The documentation of the medicinal herbal plants will be a good reference to all the young researches to carry out various conservation works.

Keywords: Ethnobotany, Manar beat, Irulas, Medicinal plants, International Union for Conservation of Nature.

(C) 2019 The Authors. Published by Innovare Academic Sciences Pvt Ltd. This is an open access article under the CC BY license (http://creativecommons. org/licenses/by/4. 0/) DOI: http://dx.doi.org/10.22159/ajpcr.2019.v12i9.34562

\section{INTRODUCTION}

The traditional knowledge in the use of plant species is a routine practice in rich diversified countries, India is one of the leading countries in this practice with heritage of cultural traditions [1]. Starting from the ancient time, the medicinal herbs play key source of drugs. According to the WHO, the world's large population relies on the traditional systems of medicines, particularly on plant-based system to meet their primary health-care needs [2]. Globally estimated that 300,000 plant species are exist, for this only around $15 \%$ have been evaluated to determine their pharmacological potential, so invention of new products from natural sources is nowadays highly encouraged [3]. Some of the important medicinal plants are commercially harvested for the extraction of various types of active ingredients. The various medical traditional systems such as Unani, Siddha, and Ayurvedic are hugely depended on the active medicinal properties of plants, whereas the precious wealth of indigenous knowledge is in danger of being lost. The use of traditional tribe's knowledge also reflects the values embedded in the tradition subheld by elders, especially about traditional medicine. The landscape is an essential to the efficacy of medicines, which is well understood by the practitioners, it should not be seen as "miracle" cures based on chemical compounds, but due to curative energy that draws its medicinal qualities founded on a relationship between the plants and the people [4].

Tribal communities are found dispersed in almost all states of India. There are 533 ethnic communities numbering 51,628,638 tribal people distributed all over India. Conventionally, ethnic people are highly knowledgeable about the medicinal plants and their medicinal values. These indigenous people are using a historical continuity of resource use and possess a broad knowledge base of the complex ecological system in their own localities. This knowledge has been accumulated through a series of observation transmitted from one generation to next generation [5].
The conservation and sustainable utilization of biological resources are achieved through documentation of the indigenous knowledge through ethnobotanical studies [6]. The key threats for medicinally important plants are due to overdependency by local people, grazing, forest fires, and commercial activities. The local people depend on these plants are due to the effective nature, non-availability of medical facilities, and ethnocultural beliefs. Cultivation is clearly a sustainable alternative to the present collection of medicinal plants from the wild habitat [7].

Based on the above concepts, an extensive ethnobotanical survey was carried out in Manar beat, Karamadai range, to document the information about the traditional medicinal practices based on the medicinal plant species. The aim of the present study is to evaluate the traditional uses of local native plants to provide safe and efficient information gathered from Irulas, a local tribe inhabited in our study area and documentation of native and active plant species used for the treatment and prevention of various diseases and ailments.

\section{METHODS}

\section{Study area}

Ethnobotanical survey was carried out in Manar beat, Karamadai range, Western Ghats, Tamil Nadu, from January 2018 to December 2018. The study area lies between $11^{\circ} 16^{\prime} \mathrm{N}$ latitude and $76^{\circ} 58^{\prime} \mathrm{E}$ longitude. It has tropical climate with maximum temperature beyond $35^{\circ} \mathrm{C}$ during summer (May-June) and below $21^{\circ} \mathrm{C}$ during winter (DecemberJanuary) and average annual rainfall is about $709 \mathrm{~mm}$. Different types of vegetation are available in Karamadai reserve forest, namely, scrub jungle, dry deciduous forest, mixed deciduous forest, moist deciduous forest, and riparian vegetation. Manar beat is an evergreen tropical moist deciduous forest with rich vegetation of floras and faunas. The 
Table 1: Summary of surveyed medicinal plants in Mannar beat, Karamadai range, Western Ghats

\begin{tabular}{|c|c|c|c|c|c|c|c|c|}
\hline S. No. & Botanical name & Family name & Local name & Habit & $\begin{array}{l}\text { Parts } \\
\text { used }\end{array}$ & Active principle & Therapeutic uses & $\begin{array}{l}\text { Mode of } \\
\text { preparation }\end{array}$ \\
\hline 1. & $\begin{array}{l}\text { Acacia caesia } \\
\text { Willd. }\end{array}$ & Mimosaceae & Nanjupattai & $\begin{array}{l}\text { Climbing } \\
\text { shrub }\end{array}$ & Bark & Phenols & $\begin{array}{l}\text { Wound healing and } \\
\text { skin diseases }\end{array}$ & Paste \\
\hline 2. & $\begin{array}{l}\text { Acacia concinna } \\
\text { Dc. }\end{array}$ & Mimosaceae & Shiakakai & $\begin{array}{l}\text { Climbing } \\
\text { shrub }\end{array}$ & $\begin{array}{l}\text { Bark, } \\
\text { leaves }\end{array}$ & Alkaloids & $\begin{array}{l}\text { Jaundice, } \\
\text { constipation, skin } \\
\text { problems, and } \\
\text { astringent }\end{array}$ & Powder \\
\hline 4. & $\begin{array}{l}\text { Acanthus ilicifolius } \\
\text { L. }\end{array}$ & Acanthaceae & Kalutai mulli & Shrub & $\begin{array}{l}\text { Roots, } \\
\text { leaves, } \\
\text { and stem }\end{array}$ & $\begin{array}{l}\text { Steroids and } \\
\text { terpenoids }\end{array}$ & $\begin{array}{l}\text { Rheumatism, } \\
\text { asthma, paralysis, } \\
\text { psoriasis, astringent, } \\
\text { wounds, and } \\
\text { leukorrhea }\end{array}$ & Decoction \\
\hline 6. & $\begin{array}{l}\text { Adenanthera } \\
\text { pavonina L. }\end{array}$ & Mimosaceae & Ani kundumani & Tree & Leaves & Fatty acids & Diarrhea & Juice \\
\hline 7. & $\begin{array}{l}\text { Adenostemma } \\
\text { lavenia } 0 . \mathrm{Kze} .\end{array}$ & Asteraceae & Vadakala & Herb & $\begin{array}{l}\text { Leaves } \\
\text { and root }\end{array}$ & Alkaloids & $\begin{array}{l}\text { Wound healing, } \\
\text { injuries, and worms }\end{array}$ & $\begin{array}{l}\text { Extraction } \\
\text { and paste }\end{array}$ \\
\hline 8. & $\begin{array}{l}\text { Aerides maculosum } \\
\text { Lindl. }\end{array}$ & Orchidaceae & - & Epiphyte & $\begin{array}{l}\text { Leaves } \\
\text { and } \\
\text { flower }\end{array}$ & $\begin{array}{l}\text { Glycosides, } \\
\text { saponins, and } \\
\text { steroids }\end{array}$ & $\begin{array}{l}\text { Skin diseases and } \\
\text { wound healing }\end{array}$ & Decoction \\
\hline 9. & Aerva lanata Juss. & Amaranthaceae & Ciru-pulai & Herb & Roots & Flavonoids & $\begin{array}{l}\text { Snakebite, cough, } \\
\text { asthma, and } \\
\text { headache }\end{array}$ & Decoction \\
\hline 10. & $\begin{array}{l}\text { Ailanthus excelsa } \\
\text { Roxb. }\end{array}$ & Simaroubaceae & Peru & Tree & Bark & $\begin{array}{l}\text { Alkaloids and } \\
\text { flavonoids }\end{array}$ & $\begin{array}{l}\text { Skin diseases, } \\
\text { jaundice, } \\
\text { anthelmintic, } \\
\text { expectorant, } \\
\text { antiasthmatic, } \\
\text { allergy, } \\
\text { antispasmodic, } \\
\text { antipyretic, and } \\
\text { bronchoconstriction }\end{array}$ & Paste \\
\hline 11. & $\begin{array}{l}\text { Alangium } \\
\text { salvifolium Wang. }\end{array}$ & Alangiaceae & Alandi & Tree & $\begin{array}{l}\text { Root, } \\
\text { seeds, } \\
\text { fruits, } \\
\text { and } \\
\text { leaves }\end{array}$ & $\begin{array}{l}\text { Flavonoids, } \\
\text { glycosides }\end{array}$ & $\begin{array}{l}\text { Hemorrhoids, } \\
\text { rheumatism } \\
\text { arthritis, loose stool, } \\
\text { herpes, and blood } \\
\text { disorders }\end{array}$ & $\begin{array}{l}\text { Decoction } \\
\text { and paste }\end{array}$ \\
\hline 12. & $\begin{array}{l}\text { Albizzia amara } \\
\text { Boiv. }\end{array}$ & Mimosaceae & Oonjapattai & Tree & $\begin{array}{l}\text { Bark and } \\
\text { root }\end{array}$ & $\begin{array}{l}\text { Terpenoids, } \\
\text { saponins }\end{array}$ & $\begin{array}{l}\text { Inflammations and } \\
\text { snakebite }\end{array}$ & Decoction \\
\hline 13. & $\begin{array}{l}\text { Alysicarpus } \\
\text { monilifer DC. }\end{array}$ & Fabaceae & Kasukkoti & Herb & $\begin{array}{l}\text { Leaves, } \\
\text { stem, and } \\
\text { root }\end{array}$ & $\begin{array}{l}\text { Saponins and } \\
\text { alkaloids }\end{array}$ & $\begin{array}{l}\text { Inflammation, chest } \\
\text { pain, skin diseases, } \\
\text { jaundice, and fever }\end{array}$ & $\begin{array}{l}\text { Paste and } \\
\text { decoction }\end{array}$ \\
\hline 14. & $\begin{array}{l}\text { Anisomeles } \\
\text { malabarica } \mathrm{R} . \mathrm{Br} .\end{array}$ & Lamiaceae & Payemiratti & Herb & $\begin{array}{l}\text { Whole } \\
\text { plant and } \\
\text { leaves }\end{array}$ & $\begin{array}{l}\text { Alkaloids and } \\
\text { glycosides }\end{array}$ & $\begin{array}{l}\text { Antispasmodic, } \\
\text { diaphoretic, } \\
\text { rheumatic pains, } \\
\text { dyspepsia, and colic }\end{array}$ & Paste \\
\hline 15. & $\begin{array}{l}\text { Anodendron } \\
\text { paniculatum A. DC. }\end{array}$ & Apocynaceae & Sarakkodi & Climber & $\begin{array}{l}\text { Leaves } \\
\text { and fruits }\end{array}$ & $\begin{array}{l}\text { Alkaloids and } \\
\text { glycosides }\end{array}$ & Jaundice & Powder \\
\hline 16. & $\begin{array}{l}\text { Argyreia cuneata } \\
\text { Ker Gawl. }\end{array}$ & Convolvulaceae & Kanvalipoo & $\begin{array}{l}\text { Climbing } \\
\text { shrub }\end{array}$ & Leaves & $\begin{array}{l}\text { Alkaloids and } \\
\text { lipids }\end{array}$ & $\begin{array}{l}\text { Diabetes, skin } \\
\text { diseases, and cough }\end{array}$ & Decoction \\
\hline 17. & $\begin{array}{l}\text { Aristolochia indica } \\
\text { Linn. }\end{array}$ & Aristolochiaceae & - & Climber & $\begin{array}{l}\text { Whole } \\
\text { plant }\end{array}$ & $\begin{array}{l}\text { Alkaloids and } \\
\text { flavonoids }\end{array}$ & $\begin{array}{l}\text { Cough, astringent, } \\
\text { and purgative }\end{array}$ & Juice \\
\hline 18. & $\begin{array}{l}\text { Artocarpus hirsuta } \\
\text { Lamk. }\end{array}$ & Moraceae & Aiyinipila & Tree & $\begin{array}{l}\text { Seed and } \\
\text { fruit }\end{array}$ & Flavonoids & $\begin{array}{l}\text { Asthma and skin } \\
\text { diseases }\end{array}$ & Powder \\
\hline 19. & $\begin{array}{l}\text { Artocarpus } \\
\text { integrifolia Linn. }\end{array}$ & Moraceae & Palamarum & Tree & Root bark & $\begin{array}{l}\text { Flavonoids, } \\
\text { terpenoids }\end{array}$ & $\begin{array}{l}\text { Asthma, fever, and } \\
\text { diarrhea }\end{array}$ & Extraction \\
\hline 20. & $\begin{array}{l}\text { Atalantia } \\
\text { monophylla } \\
\text { Correa. }\end{array}$ & Rutaceae & Kattuelumeachi & Shrub & $\begin{array}{l}\text { Whole } \\
\text { plant }\end{array}$ & Alkaloids & $\begin{array}{l}\text { Rheumatism, } \\
\text { joint pains, and } \\
\text { connective tissues } \\
\text { disorders }\end{array}$ & Decoction \\
\hline
\end{tabular}


Table 1: (Continued)

\begin{tabular}{|c|c|c|c|c|c|c|c|c|}
\hline S. No. & Botanical name & Family name & Local name & Habit & $\begin{array}{l}\text { Parts } \\
\text { used }\end{array}$ & Active principle & Therapeutic uses & $\begin{array}{l}\text { Mode of } \\
\text { preparation }\end{array}$ \\
\hline 21. & $\begin{array}{l}\text { Azadirachta indica } \\
\text { A. Juss. }\end{array}$ & Meliaceae & Vembu & Tree & $\begin{array}{l}\text { Whole } \\
\text { plant }\end{array}$ & Flavonoids & $\begin{array}{l}\text { Virus infection, } \\
\text { anti-inflammatory, } \\
\text { insecticide, and skin } \\
\text { diseases }\end{array}$ & Extraction \\
\hline 22. & $\begin{array}{l}\text { Bachanania } \\
\text { axillaris (Desr.) }\end{array}$ & Anacardiaceae & Kolamaavu & Tree & $\begin{array}{l}\text { Bark, } \\
\text { fruit, and } \\
\text { leaves }\end{array}$ & $\begin{array}{l}\text { Phenols and } \\
\text { flavonoids }\end{array}$ & $\begin{array}{l}\text { Anticancer, } \\
\text { antidiarrheal, } \\
\text { anti-inflammatory, } \\
\text { antioxidant, } \\
\text { depurative, } \\
\text { purgative, and tonic }\end{array}$ & Decoction \\
\hline 23. & $\begin{array}{l}\text { Bauhinia racemosa } \\
\text { Lamk. }\end{array}$ & Fabaceae & Vellaimantarai & Tree & $\begin{array}{l}\text { Whole } \\
\text { plant }\end{array}$ & $\begin{array}{l}\text { Phenols and } \\
\text { flavonoids }\end{array}$ & $\begin{array}{l}\text { Cough, abdominal } \\
\text { diseases, anorexia, } \\
\text { and ascaris }\end{array}$ & $\begin{array}{l}\text { Juice and } \\
\text { decoction }\end{array}$ \\
\hline 24. & $\begin{array}{l}\text { Begonia } \\
\text { malabarica Lamk. }\end{array}$ & Begoniaceae & - & Herb & $\begin{array}{l}\text { Leaves } \\
\text { and } \\
\text { whole } \\
\text { plant }\end{array}$ & $\begin{array}{l}\text { Flavonoids and } \\
\text { steroids }\end{array}$ & $\begin{array}{l}\text { Respiratory } \\
\text { infections, diarrhea, } \\
\text { blood cancer, and } \\
\text { skin diseases }\end{array}$ & $\begin{array}{l}\text { Decoction } \\
\text { and paste }\end{array}$ \\
\hline 25. & $\begin{array}{l}\text { Benkara } \\
\text { malabarica Lamk. } \\
\text { Tirveng. }\end{array}$ & Rubiaceae & Sirukarai & $\begin{array}{l}\text { Thorny } \\
\text { small } \\
\text { tree }\end{array}$ & Leaves & $\begin{array}{l}\text { Alkaloids and } \\
\text { flavonoids }\end{array}$ & $\begin{array}{l}\text { Abdominal pain and } \\
\text { throat infection }\end{array}$ & $\begin{array}{l}\text { Juice and } \\
\text { paste }\end{array}$ \\
\hline 26. & $\begin{array}{l}\text { Blachia umbellata } \\
\text { Baill. }\end{array}$ & Euphorbiaceae & Aatthumanthai & Shrub & Leaves & & Rheumatism & $\begin{array}{l}\text { Paste and } \\
\text { tonic }\end{array}$ \\
\hline 27. & $\begin{array}{l}\text { Blepharis } \\
\text { boerhaaviaefolia } \\
\text { Pers. }\end{array}$ & Acanthaceae & - & $\begin{array}{l}\text { Under } \\
\text { shrub }\end{array}$ & $\begin{array}{l}\text { Leaves, } \\
\text { root, } \\
\text { fruit, and } \\
\text { seeds }\end{array}$ & $\begin{array}{l}\text { Alkaloids and } \\
\text { flavonoids }\end{array}$ & $\begin{array}{l}\text { Wound healing, } \\
\text { ulcers, nasal, } \\
\text { asthma, throat } \\
\text { inflammation, spleen } \\
\text { disorders, diarrhea, } \\
\text { urinary disorder, } \\
\text { kidney stone, and } \\
\text { nervous disorders }\end{array}$ & Decoction \\
\hline 28. & $\begin{array}{l}\text { Cadaba } \\
\text { fruticosa (L.) } \\
\text { Druce. }\end{array}$ & Capparidaceae & Chikondai & Shrub & Leaves & $\begin{array}{l}\text { Alkaloids and } \\
\text { glycosides }\end{array}$ & $\begin{array}{l}\text { Dysentery, diarrhea, } \\
\text { body pain, and } \\
\text { poisonous bites }\end{array}$ & Juice \\
\hline 29. & $\begin{array}{l}\text { Cadaba trifoliata } \\
\text { Wight. \& Arn. }\end{array}$ & Capparidaceae & Kattagatti & Shrub & $\begin{array}{l}\text { Leaves, } \\
\text { stem, and } \\
\text { roots }\end{array}$ & Tannins & $\begin{array}{l}\text { Antirheumatic, } \\
\text { anthelmintic, } \\
\text { antibacterial, and } \\
\text { viral infection }\end{array}$ & $\begin{array}{l}\text { Decoction } \\
\text { and } \\
\text { extraction }\end{array}$ \\
\hline 30. & $\begin{array}{l}\text { Calamus rotang } \\
\text { Linn. }\end{array}$ & Arecaceae & Pirambu & Climber & $\begin{array}{l}\text { Fruit and } \\
\text { leaves }\end{array}$ & Flavonoids & $\begin{array}{l}\text { Astringent, } \\
\text { antidiarrheal, } \\
\text { anti-inflammatory, } \\
\text { chronic fevers, } \\
\text { piles, abdominal } \\
\text { tumors, strangury, } \\
\text { antibilious, and } \\
\text { spasmolytic }\end{array}$ & Decoction \\
\hline 31. & $\begin{array}{l}\text { Capparis grandis } \\
\text { Linn. f. }\end{array}$ & Capparidaceae & Pachara & Tree & $\begin{array}{l}\text { Whole } \\
\text { plant }\end{array}$ & $\begin{array}{l}\text { Alkaloids and } \\
\text { flavonoids }\end{array}$ & $\begin{array}{l}\text { Ulcer, asthma, and } \\
\text { anorexia }\end{array}$ & $\begin{array}{l}\text { Tonic and } \\
\text { juice }\end{array}$ \\
\hline 32. & $\begin{array}{l}\text { Capparis zeylanica } \\
\text { Linn. }\end{array}$ & Capparidaceae & Adhandai & Shrub & Root & $\begin{array}{l}\text { Fatty acids and } \\
\text { flavonoids }\end{array}$ & $\begin{array}{l}\text { Dysentery and } \\
\text { diarrhea }\end{array}$ & Extraction \\
\hline 33. & $\begin{array}{l}\text { Caralluma } \\
\text { adscendens R.Br. }\end{array}$ & Asclepiadaceae & Kallimudayan & Herb & $\begin{array}{l}\text { Stem, } \\
\text { root, and } \\
\text { flower }\end{array}$ & Lipids & $\begin{array}{l}\text { Cough, cold, } \\
\text { diarrhea, high } \\
\text { pressure, and } \\
\text { swelling }\end{array}$ & $\begin{array}{l}\text { Decoction } \\
\text { and paste }\end{array}$ \\
\hline 34. & $\begin{array}{l}\text { Caralluma } \\
\text { pauciflora N. E.Br. }\end{array}$ & Asclepiadaceae & Puliyanprinadai & Herb & $\begin{array}{l}\text { Leaves } \\
\text { and } \\
\text { whole } \\
\text { plant }\end{array}$ & $\begin{array}{l}\text { Flavonoids and } \\
\text { saponins }\end{array}$ & $\begin{array}{l}\text { Ulcer, rheumatism, } \\
\text { diabetes, and } \\
\text { inflammation }\end{array}$ & $\begin{array}{l}\text { Decoction } \\
\text { and paste }\end{array}$ \\
\hline 35. & $\begin{array}{l}\text { Caralluma } \\
\text { umbellata Haw. }\end{array}$ & Asclepiadaceae & $\begin{array}{l}\text { Erumaikalli } \\
\text { mulayan }\end{array}$ & Herb & Stem & Glycosides & $\begin{array}{l}\text { Stomach disorder, } \\
\text { abdominal pain, } \\
\text { obesity, diabetes, } \\
\text { and ulcer problems }\end{array}$ & Juice \\
\hline 36. & Cassia javanica L. & Caesalpiniaceae & Konari & Tree & $\begin{array}{l}\text { Seeds } \\
\text { and bark }\end{array}$ & $\begin{array}{l}\text { Glycosides and } \\
\text { flavonoids }\end{array}$ & $\begin{array}{l}\text { Laxative, antipyretic, } \\
\text { fever, and emesis }\end{array}$ & Decoction \\
\hline
\end{tabular}


Table 1: (Continued)

\begin{tabular}{|c|c|c|c|c|c|c|c|c|}
\hline S. No. & Botanical name & Family name & Local name & Habit & $\begin{array}{l}\text { Parts } \\
\text { used }\end{array}$ & Active principle & Therapeutic uses & $\begin{array}{l}\text { Mode of } \\
\text { preparation }\end{array}$ \\
\hline 37. & $\begin{array}{l}\text { Cassia occidentalis } \\
\text { Linn. }\end{array}$ & Caesalpiniaceae & Peyaverai & Shrub & $\begin{array}{l}\text { Seeds, } \\
\text { leaves, } \\
\text { root, } \\
\text { fruit, and } \\
\text { whole } \\
\text { plant }\end{array}$ & Glycosides & $\begin{array}{l}\text { Cutaneous diseases, } \\
\text { cough, asthma, } \\
\text { sweetish, bitter, } \\
\text { stomachic, fevers, } \\
\text { good for sore } \\
\text { throat, diuretic, } \\
\text { ringworm, scorpion, } \\
\text { elephantiasis, sting, } \\
\text { snakebite, ascites, } \\
\text { purgative, febrifuge, } \\
\text { sore eyes, and skin } \\
\text { diseases }\end{array}$ & Extraction \\
\hline 38. & $\begin{array}{l}\text { Celtis phillipensis } \\
\text { Blanco. }\end{array}$ & Cannabaceae & Kodalimuruki & Tree & Root & Terpenoids & Diarrhea & Decoction \\
\hline 39. & $\begin{array}{l}\text { Cenchrus ciliaris } \\
\text { Linn. }\end{array}$ & Poaceae & Kollukattai pullu & Herb & Leaves & Lipids & $\begin{array}{l}\text { Kidney pain, wound } \\
\text { healing, and tumors }\end{array}$ & Decoction \\
\hline 40. & $\begin{array}{l}\text { Centella asiatica } \\
\text { Urb. }\end{array}$ & Apiaceae & Vallarai & $\begin{array}{l}\text { Creeping } \\
\text { herb }\end{array}$ & Leaves & $\begin{array}{l}\text { Terpenoids and } \\
\text { glycosides }\end{array}$ & $\begin{array}{l}\text { Wound healing, } \\
\text { brain tonic, and } \\
\text { cardiotonic }\end{array}$ & Infusion \\
\hline 41. & $\begin{array}{l}\text { Cereus pterogonus } \\
\text { Lamk. }\end{array}$ & Cactaceae & Ooci kalli & Shrub & $\begin{array}{l}\text { Whole } \\
\text { plant }\end{array}$ & Proteins & $\begin{array}{l}\text { Purgative, } \\
\text { astringent, } \\
\text { constipation, } \\
\text { refrigerant, } \\
\text { antiperiodic, and } \\
\text { antipyretic }\end{array}$ & Extraction \\
\hline 42. & $\begin{array}{l}\text { Ceropegia juncea } \\
\text { Roxb. }\end{array}$ & Asclepiadaceae & Jaathili & $\begin{array}{l}\text { Climbing } \\
\text { herb }\end{array}$ & $\begin{array}{l}\text { Leaves } \\
\text { and root }\end{array}$ & $\begin{array}{l}\text { Alkaloids and } \\
\text { steroids }\end{array}$ & $\begin{array}{l}\text { Bacterial infection, } \\
\text { ulcer, and } \\
\text { inflammation }\end{array}$ & $\begin{array}{l}\text { Decoction } \\
\text { and juice }\end{array}$ \\
\hline 43. & $\begin{array}{l}\text { Chamaecrista } \\
\text { pumila (Lam.) K. } \\
\text { Larsen. }\end{array}$ & Fabaceae & - & Shrub & $\begin{array}{l}\text { Whole } \\
\text { plant and } \\
\text { leaves }\end{array}$ & $\begin{array}{l}\text { Tannins and } \\
\text { flavonoids }\end{array}$ & $\begin{array}{l}\text { Diarrhea and } \\
\text { bacterial infection }\end{array}$ & Decoction \\
\hline 44. & $\begin{array}{l}\text { Cipadessa } \\
\text { baccifera Miq. }\end{array}$ & Meliaceae & Pullipancheddi & Shrub & $\begin{array}{l}\text { Whole } \\
\text { plant }\end{array}$ & Alkaloids & $\begin{array}{l}\text { Indigestion, cough, } \\
\text { and antifertility }\end{array}$ & Juice \\
\hline 45. & $\begin{array}{l}\text { Cissampelos } \\
\text { pareira Linn. }\end{array}$ & Menispermaceae & $\begin{array}{l}\text { Malai } \\
\text { Thangivaer }\end{array}$ & Climber & $\begin{array}{l}\text { Root and } \\
\text { leaves }\end{array}$ & $\begin{array}{l}\text { Flavonoids and } \\
\text { alkaloids }\end{array}$ & $\begin{array}{l}\text { Wound healing, } \\
\text { antidote, anorexia, } \\
\text { indigestion, blood } \\
\text { purification, and } \\
\text { anti-inflammation }\end{array}$ & Paste \\
\hline 46. & $\begin{array}{l}\text { Cissus } \\
\text { quadrangularis } \\
\text { Linn. }\end{array}$ & Vitaceae & Pirandai & $\begin{array}{l}\text { Climbing } \\
\text { shrub }\end{array}$ & $\begin{array}{l}\text { Stem, } \\
\text { root, and } \\
\text { leaves }\end{array}$ & $\begin{array}{l}\text { Flavonoids and } \\
\text { terpenoids }\end{array}$ & $\begin{array}{l}\text { Bone breakage, } \\
\text { appetizer dyspepsia, } \\
\text { indigestion, and } \\
\text { piles }\end{array}$ & Juice \\
\hline 47. & $\begin{array}{l}\text { Clausena } \\
\text { dentata (Willd.) M. } \\
\text { Roem. }\end{array}$ & Rutaceae & $\begin{array}{l}\text { Kattu } \\
\text { karuveppilai }\end{array}$ & $\begin{array}{l}\text { Small } \\
\text { tree }\end{array}$ & $\begin{array}{l}\text { Leaves } \\
\text { and root }\end{array}$ & $\begin{array}{l}\text { Alkaloids and } \\
\text { coumarins }\end{array}$ & $\begin{array}{l}\text { Gastrointestinal } \\
\text { disorders, fever, } \\
\text { rheumatism, } \\
\text { headache, } \\
\text { hypotension, and } \\
\text { sore throat }\end{array}$ & $\begin{array}{l}\text { Tonic and } \\
\text { paste }\end{array}$ \\
\hline 48. & $\begin{array}{l}\text { Clerodendron } \\
\text { serratum Spr. }\end{array}$ & Verbenaceae & Angaravalli & Shrub & $\begin{array}{l}\text { Leaves, } \\
\text { stem, } \\
\text { seed, and } \\
\text { root }\end{array}$ & $\begin{array}{l}\text { Flavonoids and } \\
\text { phenols }\end{array}$ & $\begin{array}{l}\text { Asthma and } \\
\text { respiratory diseases }\end{array}$ & $\begin{array}{l}\text { Paste and } \\
\text { decoction }\end{array}$ \\
\hline 49. & $\begin{array}{l}\text { Coccinia } \\
\text { grandis (Linn.) } \\
\text { Voigt. }\end{array}$ & Cucurbitaceae & Kovakai & Climber & Fruit & $\begin{array}{l}\text { Alkaloids and } \\
\text { glycosides }\end{array}$ & $\begin{array}{l}\text { Leprosy, fever, } \\
\text { asthma, bronchitis, } \\
\text { and jaundice }\end{array}$ & Juice \\
\hline 50. & $\begin{array}{l}\text { Combretum } \\
\text { albidum G. Don. }\end{array}$ & Combretaceae & Odai Kodi & Climber & $\begin{array}{l}\text { Leaves, } \\
\text { fruit, } \\
\text { and stem } \\
\text { bark }\end{array}$ & $\begin{array}{l}\text { Terpenoids and } \\
\text { flavonoids }\end{array}$ & $\begin{array}{l}\text { Peptic ulcer, } \\
\text { diarrhea, dysentery, } \\
\text { jaundice, and skin } \\
\text { diseases }\end{array}$ & $\begin{array}{l}\text { Paste, juice, } \\
\text { and decoction }\end{array}$ \\
\hline 51. & $\begin{array}{l}\text { Cordia sinensis } \\
\text { Lam. }\end{array}$ & Boraginaceae & Sellai & $\begin{array}{l}\text { Small } \\
\text { tree }\end{array}$ & $\begin{array}{l}\text { Leaves } \\
\text { and fruit }\end{array}$ & Flavonoids & $\begin{array}{l}\text { Anti-inflammatory, } \\
\text { blood pressure, } \\
\text { hypotensive, and } \\
\text { diuretic }\end{array}$ & Decoction \\
\hline 52. & $\begin{array}{l}\text { Crataeva adansonii } \\
\text { DC. }\end{array}$ & Capparidaceae & Marvilinga & $\begin{array}{l}\text { Small } \\
\text { tree }\end{array}$ & $\begin{array}{l}\text { Stem } \\
\text { bark }\end{array}$ & Phenols & Joint pain & Decoction \\
\hline
\end{tabular}


Table 1: (Continued)

\begin{tabular}{|c|c|c|c|c|c|c|c|c|}
\hline S. No. & Botanical name & Family name & Local name & Habit & $\begin{array}{l}\text { Parts } \\
\text { used }\end{array}$ & Active principle & Therapeutic uses & $\begin{array}{l}\text { Mode of } \\
\text { preparation }\end{array}$ \\
\hline 53. & $\begin{array}{l}\text { Crataeva religiosa } \\
\text { Forst. }\end{array}$ & Capparidaceae & Mavilankai & $\begin{array}{l}\text { Small } \\
\text { tree }\end{array}$ & Bark & $\begin{array}{l}\text { Phenols and } \\
\text { terpenoids }\end{array}$ & $\begin{array}{l}\text { Urinary complaints, } \\
\text { snakebite, and } \\
\text { ascites }\end{array}$ & Decoction \\
\hline 54. & $\begin{array}{l}\text { Crotalaria } \\
\text { hebecarpa (DC.) } \\
\text { Rudd. }\end{array}$ & Fabaceae & Godhadi & Herb & $\begin{array}{l}\text { Whole } \\
\text { plant }\end{array}$ & Flavonoids & $\begin{array}{l}\text { Skin diseases, } \\
\text { snakebites, and } \\
\text { jaundice }\end{array}$ & $\begin{array}{l}\text { Paste and } \\
\text { powder }\end{array}$ \\
\hline 55. & $\begin{array}{l}\text { Crotalaria pallida } \\
\text { Aiton. }\end{array}$ & Fabaceae & Kilukiluppai & Shrub & Leaves & $\begin{array}{l}\text { Alkaloids and } \\
\text { flavonoids }\end{array}$ & Vermifuge and fever & Extraction \\
\hline 56. & $\begin{array}{l}\text { Cyrtococcum } \\
\text { patens A. Cam. }\end{array}$ & Poaceae & - & Herb & Leaves & Alkaloids & $\begin{array}{l}\text { Fever, cough, and } \\
\text { anti-inflammation }\end{array}$ & Decoction \\
\hline 57. & $\begin{array}{l}\text { Cyrtococcum } \\
\text { trigonum A. Cam. }\end{array}$ & Poaceae & Abbu karkai & Herb & $\begin{array}{l}\text { Leaves } \\
\text { and root }\end{array}$ & Alkaloids & Nervous disorder & $\begin{array}{l}\text { Paste and } \\
\text { decoction }\end{array}$ \\
\hline 58. & $\begin{array}{l}\text { Daemia extensa } \\
\text { R.Br. }\end{array}$ & Asclepiadaceae & Kodalma & Climber & $\begin{array}{l}\text { Whole } \\
\text { plant }\end{array}$ & $\begin{array}{l}\text { Saponins and } \\
\text { tannins }\end{array}$ & $\begin{array}{l}\text { Gastric ulcers, } \\
\text { uterine, and } \\
\text { menstrual } \\
\text { complaints }\end{array}$ & $\begin{array}{l}\text { Juice and } \\
\text { decoction }\end{array}$ \\
\hline 59. & $\begin{array}{l}\text { Dalbergia } \\
\text { coromandeliana } \\
\text { Prain. }\end{array}$ & Fabaceae & Nukkam & Shrub & $\begin{array}{l}\text { Leaves, } \\
\text { bark, and } \\
\text { fruit }\end{array}$ & $\begin{array}{l}\text { Alkaloids and } \\
\text { saponins }\end{array}$ & $\begin{array}{l}\text { Wound healing and } \\
\text { skin diseases }\end{array}$ & $\begin{array}{l}\text { Decoction } \\
\text { and paste }\end{array}$ \\
\hline 60. & $\begin{array}{l}\text { Dalbergia } \\
\text { lanceolaria Linn. f. }\end{array}$ & Fabaceae & Erigai & Tree & $\begin{array}{l}\text { Seeds, } \\
\text { root, and } \\
\text { leaves }\end{array}$ & $\begin{array}{l}\text { Phenols and } \\
\text { flavonoids }\end{array}$ & $\begin{array}{l}\text { Mild laxatives and } \\
\text { inflammatory }\end{array}$ & $\begin{array}{l}\text { Tonic and } \\
\text { juice }\end{array}$ \\
\hline 61. & $\begin{array}{l}\text { Dioscorea hirsuta } \\
\text { Blume }\end{array}$ & Dioscoreaceae & Pulidumpa & Climber & $\begin{array}{l}\text { Leaves } \\
\text { and stem }\end{array}$ & Saponins & $\begin{array}{l}\text { Diuretic, } \\
\text { rheumatism, and } \\
\text { snakebites }\end{array}$ & $\begin{array}{l}\text { Decoction, } \\
\text { juice, and } \\
\text { paste }\end{array}$ \\
\hline 62. & $\begin{array}{l}\text { Dioscorea } \\
\text { oppositifolia Linn. }\end{array}$ & Dioscoreaceae & Kavala-kodi & Climber & Tuber & Saponins & $\begin{array}{l}\text { Stomach pain, } \\
\text { spleen disorders, } \\
\text { and cancer of uterus }\end{array}$ & Decoction \\
\hline 63. & $\begin{array}{l}\text { Diospyros } \\
\text { buxifolia (Blume) } \\
\text { Hiern. }\end{array}$ & Ebenaceae & Irampalai & Tree & $\begin{array}{l}\text { Leaves, } \\
\text { stem, and } \\
\text { flower }\end{array}$ & $\begin{array}{l}\text { Alkaloids and } \\
\text { flavonoids }\end{array}$ & $\begin{array}{l}\text { Antiviral, anti-HIV, } \\
\text { and indigestion }\end{array}$ & Decoction \\
\hline 65. & $\begin{array}{l}\text { Dodonaea viscosa } \\
\text { Linn. }\end{array}$ & Sapindaceae & Virali & Shrub & $\begin{array}{l}\text { Whole } \\
\text { plant }\end{array}$ & Terpenoids & $\begin{array}{l}\text { Headache and } \\
\text { wound healing }\end{array}$ & Paste \\
\hline 66. & $\begin{array}{l}\text { Drypetes } \\
\text { roxburghii (Wall.) } \\
\text { Hurus. }\end{array}$ & Euphorbiaceae & Irukoli & Tree & $\begin{array}{l}\text { Bark and } \\
\text { leaves }\end{array}$ & - & $\begin{array}{l}\text { Joint pain and } \\
\text { rheumatism }\end{array}$ & $\begin{array}{l}\text { Decoction } \\
\text { and infusion }\end{array}$ \\
\hline 67. & $\begin{array}{l}\text { Ficus bengalensis } \\
\text { Linn. }\end{array}$ & Moraceae & Aal & Tree & $\begin{array}{l}\text { Bark and } \\
\text { latex }\end{array}$ & $\begin{array}{l}\text { Steroids and } \\
\text { flavonoids }\end{array}$ & $\begin{array}{l}\text { Rheumatism, } \\
\text { dysentery, diabetes, } \\
\text { gonorrhea, and piles }\end{array}$ & Juice \\
\hline 68. & $\begin{array}{l}\text { Ficus benjamina } \\
\text { Linn. }\end{array}$ & Moraceae & Pimpri & Tree & $\begin{array}{l}\text { Whole } \\
\text { plant }\end{array}$ & Alkaloids & Ulcers and leprosy & Decoction \\
\hline 69. & $\begin{array}{l}\text { Ficus racemosa } \\
\text { Linn. }\end{array}$ & Moraceae & Atthi & Tree & $\begin{array}{l}\text { Roots } \\
\text { and fruits }\end{array}$ & $\begin{array}{l}\text { Flavonoids and } \\
\text { terpenoids }\end{array}$ & $\begin{array}{l}\text { Blood purifier and } \\
\text { laxative }\end{array}$ & Decoction \\
\hline 70. & $\begin{array}{l}\text { Ficus religiosa } \\
\text { Linn. }\end{array}$ & Moraceae & Arasu & Tree & $\begin{array}{l}\text { Bark and } \\
\text { leaves }\end{array}$ & $\begin{array}{l}\text { Phenols and } \\
\text { tannins }\end{array}$ & $\begin{array}{l}\text { Purgative, vomiting, } \\
\text { and mouth ulcer }\end{array}$ & Decoction \\
\hline 71. & $\begin{array}{l}\text { Ficus tjakela } \\
\text { Burm. }\end{array}$ & Moraceae & - & Tree & $\begin{array}{l}\text { Leaves } \\
\text { and stem }\end{array}$ & - & $\begin{array}{l}\text { Fever, cough, and } \\
\text { cold }\end{array}$ & Decoction \\
\hline 72. & $\begin{array}{l}\text { Ficus tomentosa } \\
\text { Roxb. }\end{array}$ & Moraceae & - & Tree & $\begin{array}{l}\text { Leaves } \\
\text { and bark }\end{array}$ & Phenols & $\begin{array}{l}\text { Poultice, boils, cuts, } \\
\text { and wound }\end{array}$ & $\begin{array}{l}\text { Paste and } \\
\text { crushed } \\
\text { leaves }\end{array}$ \\
\hline 73. & $\begin{array}{l}\text { Gardenia resinifera } \\
\text { Roth. }\end{array}$ & Rubiaceae & Kambipicin & Tree & $\begin{array}{l}\text { Buds and } \\
\text { leaves }\end{array}$ & Flavonoids & $\begin{array}{l}\text { Antispasmodic, } \\
\text { expectorant, } \\
\text { carminative, and } \\
\text { stimulant }\end{array}$ & Paste \\
\hline 74. & $\begin{array}{l}\text { Helicteres isora } \\
\text { Linn. }\end{array}$ & Sterculiaceae & Vadampiri & $\begin{array}{l}\text { Large } \\
\text { shrub }\end{array}$ & $\begin{array}{l}\text { Root, } \\
\text { bark, } \\
\text { and stem } \\
\text { bark }\end{array}$ & $\begin{array}{l}\text { Flavonoids and } \\
\text { terpenoids }\end{array}$ & $\begin{array}{l}\text { Expectorant, } \\
\text { demulcent, } \\
\text { astringent, intestinal } \\
\text { worms, diarrhea, } \\
\text { and dysentery }\end{array}$ & $\begin{array}{l}\text { Decoction } \\
\text { and juice }\end{array}$ \\
\hline 75. & $\begin{array}{l}\text { Heterostemma } \\
\text { tanjorense Wight. } \\
\text { and Arn. }\end{array}$ & Apocynaceae & Palakeerai & Climber & Leaves & Alkaloids & $\begin{array}{l}\text { Antiviral, } \\
\text { antibacterial, skin } \\
\text { diseases, and fever }\end{array}$ & $\begin{array}{l}\text { Paste, tonic, } \\
\text { and infusion }\end{array}$ \\
\hline
\end{tabular}


Table 1: (Continued)

\begin{tabular}{|c|c|c|c|c|c|c|c|c|}
\hline S. No. & Botanical name & Family name & Local name & Habit & $\begin{array}{l}\text { Parts } \\
\text { used }\end{array}$ & Active principle & Therapeutic uses & $\begin{array}{l}\text { Mode of } \\
\text { preparation }\end{array}$ \\
\hline 76. & $\begin{array}{l}\text { Hibiscus } \\
\text { micranthus Linn. f. }\end{array}$ & Malvaceae & Sitraamutti & Shrub & $\begin{array}{l}\text { Leaves } \\
\text { and roots }\end{array}$ & $\begin{array}{l}\text { Tannins and } \\
\text { anthraquinones }\end{array}$ & $\begin{array}{l}\text { Asthma, diuretic, } \\
\text { and febrifuge }\end{array}$ & Decoction \\
\hline 77. & $\begin{array}{l}\text { Ipomoea obscura } \\
\text { Ker-Gawl. }\end{array}$ & Convolvulaceae & Siruthaali & Climber & $\begin{array}{l}\text { Seed, } \\
\text { root, and } \\
\text { leaves }\end{array}$ & $\begin{array}{l}\text { Alkaloids and } \\
\text { steroids }\end{array}$ & $\begin{array}{l}\text { Anthelmintic, } \\
\text { diuretic, and laxative }\end{array}$ & Decoction \\
\hline 78. & $\begin{array}{l}\text { Kyllinga triceps } \\
\text { Rottb. }\end{array}$ & Cyperaceae & Veluttanirbasi & Herb & Leaves & Terpenoids & $\begin{array}{l}\text { Antidiabetes and } \\
\text { dysentery }\end{array}$ & Juice \\
\hline 79. & $\begin{array}{l}\text { Lantana camara } \\
\text { Linn. }\end{array}$ & Verbenaceae & Unnichedi & Shrub & Leaves & $\begin{array}{l}\text { Terpenoids and } \\
\text { steroids }\end{array}$ & $\begin{array}{l}\text { Anti-inflammatory, } \\
\text { antiseptic, and } \\
\text { dysentery }\end{array}$ & Juice \\
\hline 80. & $\begin{array}{l}\text { Neptunia oleracea } \\
\text { Lour. }\end{array}$ & Mimosaceae & Sundaikkirai & Herb & Root & Phenols & $\begin{array}{l}\text { Bones of the nose } \\
\text { and hard palate, } \\
\text { syphilis, and cure } \\
\text { earache }\end{array}$ & Juice \\
\hline 81. & $\begin{array}{l}\text { Oldenlandia } \\
\text { herbacea Roxb. }\end{array}$ & Rubiaceae & Nonnanampullu & Herb & $\begin{array}{l}\text { Whole } \\
\text { plant and } \\
\text { leaves }\end{array}$ & Glycosides & $\begin{array}{l}\text { Elephantiasis, } \\
\text { fever, verminosis, } \\
\text { inflammation, } \\
\text { asthma, bronchitis, } \\
\text { and ulcer }\end{array}$ & $\begin{array}{l}\text { Decoction } \\
\text { and paste }\end{array}$ \\
\hline 82. & $\begin{array}{l}\text { Perotis indica } 0 . \\
\text { Ktz. }\end{array}$ & Poaceae & Narival & Herb & $\begin{array}{l}\text { Whole } \\
\text { plant }\end{array}$ & - & $\begin{array}{l}\text { Snakebites and } \\
\text { bronchitis }\end{array}$ & Infusion \\
\hline 83. & $\begin{array}{l}\text { Phyllanthus debilis } \\
\text { Hook.f. }\end{array}$ & Euphorbiaceae & Arulundi & Tree & $\begin{array}{l}\text { Root, } \\
\text { leaves, } \\
\text { and } \\
\text { whole } \\
\text { plant }\end{array}$ & $\begin{array}{l}\text { Tannins and } \\
\text { terpenoids }\end{array}$ & $\begin{array}{l}\text { Fever, jaundice, } \\
\text { gastritis, urinary } \\
\text { difficulties, } \\
\text { bone fractures, } \\
\text { menorrhagia, } \\
\text { leukorrhea, asthma, } \\
\text { endometritis, wound } \\
\text { healing, and liver } \\
\text { diseases }\end{array}$ & $\begin{array}{l}\text { Paste and } \\
\text { decoction }\end{array}$ \\
\hline 84. & $\begin{array}{l}\text { Pongamia } \\
\text { pinnata (L.) Pierre }\end{array}$ & Fabaceae & Pungan & Tree & $\begin{array}{l}\text { Leaves, } \\
\text { stem, } \\
\text { seed, and } \\
\text { flower }\end{array}$ & Steroids & $\begin{array}{l}\text { Antidiabetic, } \\
\text { rheumatism, } \\
\text { Anti-inflammatory, } \\
\text { piles, skin diseases, } \\
\text { and wounds }\end{array}$ & $\begin{array}{l}\text { Juice and } \\
\text { paste }\end{array}$ \\
\hline 85. & $\begin{array}{l}\text { Salvadora persica } \\
\text { Linn. }\end{array}$ & Salvadoraceae & Uka & Shrub & Leaves & Flavonoids & $\begin{array}{l}\text { Antiplaque and } \\
\text { analgesic }\end{array}$ & Tonic \\
\hline 86. & $\begin{array}{l}\text { Santalum album } \\
\text { Linn. }\end{array}$ & Santalaceae & Sandhanam & Tree & $\begin{array}{l}\text { Leaves } \\
\text { and stem }\end{array}$ & Fatty oils & $\begin{array}{l}\text { Gastric irritability, } \\
\text { dysentery, skin } \\
\text { diseases, and } \\
\text { gonorrhea }\end{array}$ & Paste \\
\hline 87. & $\begin{array}{l}\text { Terminalia } \\
\text { arjuna (Roxb.) } \\
\text { Wight and Arn. }\end{array}$ & Combretaceae & Marudha maram & Tree & $\begin{array}{l}\text { Bark, } \\
\text { leaves }\end{array}$ & Flavonoids & $\begin{array}{l}\text { Heart disease, } \\
\text { ulcers, dysentery, } \\
\text { and wounds }\end{array}$ & $\begin{array}{l}\text { Decoction } \\
\text { and powder }\end{array}$ \\
\hline 88. & $\begin{array}{l}\text { Vallaris solanacea } \\
\text { O. Kze. }\end{array}$ & Apocynaceae & - & Climber & $\begin{array}{l}\text { Root and } \\
\text { bark }\end{array}$ & Terpenoids & $\begin{array}{l}\text { Analgesic, } \\
\text { antidiarrheal, and } \\
\text { dysentery }\end{array}$ & $\begin{array}{l}\text { Tonic and } \\
\text { paste }\end{array}$ \\
\hline 89. & $\begin{array}{l}\text { Ziziphus oenoplia } \\
\text { Mill. }\end{array}$ & Rhamnaceae & Churipala chedi & $\begin{array}{l}\text { Climbing } \\
\text { shrub }\end{array}$ & $\begin{array}{l}\text { Fruit and } \\
\text { bark }\end{array}$ & $\begin{array}{l}\text { Flavonoids and } \\
\text { phenols }\end{array}$ & $\begin{array}{l}\text { Diarrhea, diabetes, } \\
\text { and anti-cancerous }\end{array}$ & Decoction \\
\hline
\end{tabular}

vegetation is floristically rich compared to other regions and represents several unique habitats. The vegetation was conducted in six small villages of Manar beat situated in Karamadai range which are occupied by Irula tribals.

\section{Data collection}

Fieldwork was conducted over the 12 months period focusing on collecting ethnobotanical information from local people about the medicinal plants in Manar beat. A total of 15 tribal people (seven men and eight women) aged between 35 and 85 who were cooperating fully were interviewed. Interview was conducted using semi-structured questionnaires and open-ended conversations at homes. The vegetation of the study area, plants therapeutical properties, and the kind of ailments used were among the questions asked. All kinds of information were documented and recorded.

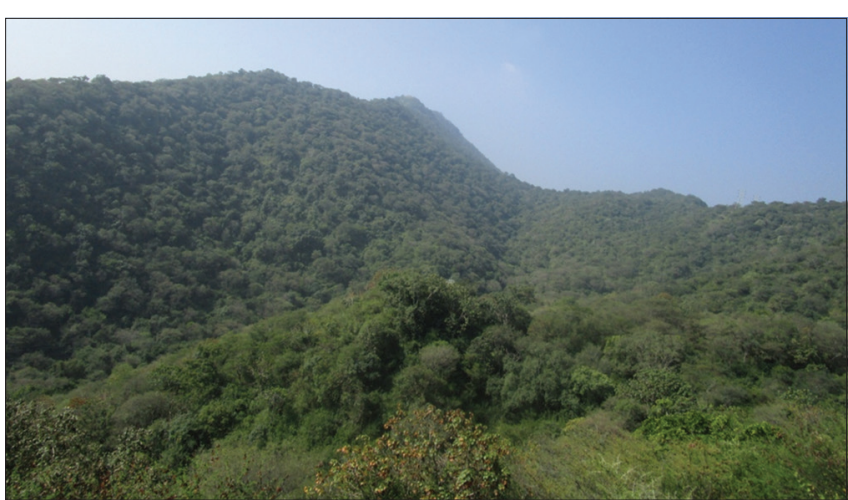

Fig. 1: Hill view of Manar beat, Karamadai range 


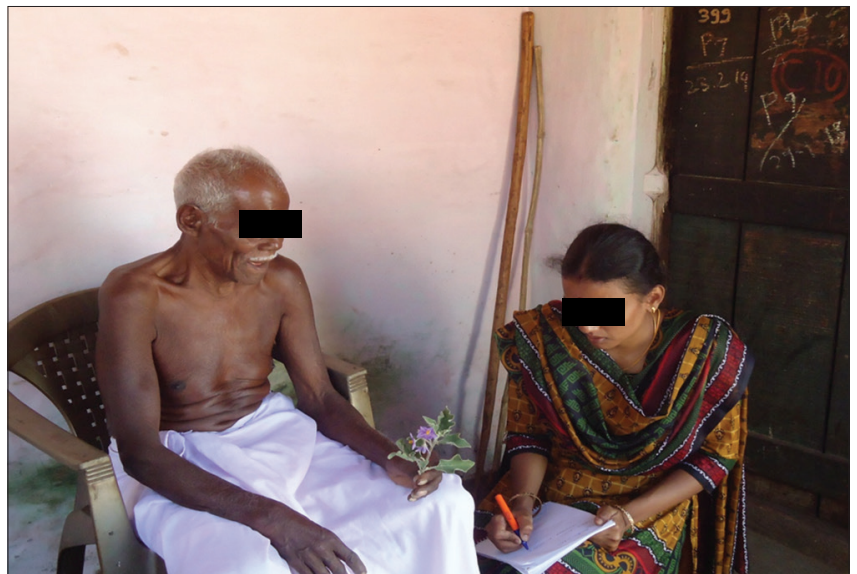

Fig. 2: Collection of traditional knowledge of plants from tribe

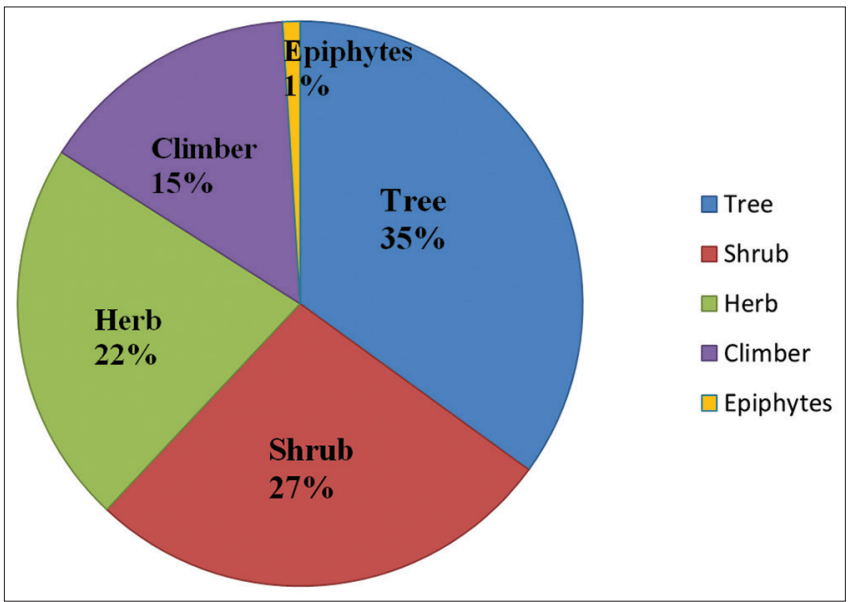

Fig. 3: Life form of plants used as medicinal plants in Manar beat

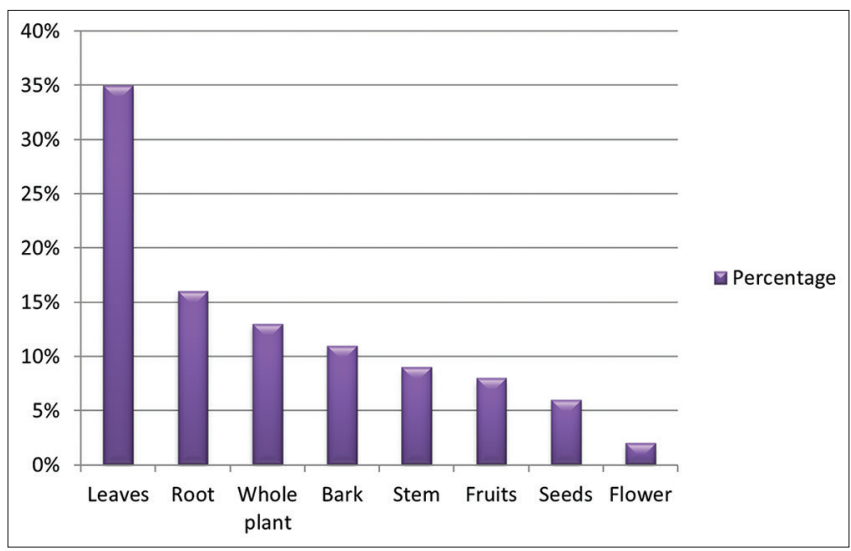

Fig. 4: Different plant parts used for the various health-care problems in Manar beat tribes

\section{Identification}

Identity of the collected plant species was done with the volumes of The Flora of the Nilgiri and Pulney Hill-tops [8], The Flora of Presidency of Madras [9], and The Flora of Tamil Nadu Carnatic [10]. The identity is authenticated by matched with type specimens available in the herbarium of Botanical Survey of India, Southern Circle, TNAU Campus, Coimbatore, Tamil Nadu. Herbarium specimens were collected and deposited in the Herbarium of Botany Department, Vellalar College for Women (Autonomous), Erode, Tamil Nadu, India, for future reference.
Table 2: Distribution of species occurred in different families

\begin{tabular}{|c|c|c|}
\hline S. No. & Name of the families & Number of the species \\
\hline 1. & Acanthaceae & 2 \\
\hline 2. & Alangiaceae & 1 \\
\hline 3. & Amaranthaceae & 2 \\
\hline 4. & Anacardiaceae & 1 \\
\hline 5. & Apiaceae & 1 \\
\hline 6. & Apocynaceae & 3 \\
\hline 7. & Arecaceae & 1 \\
\hline 8. & Aristolochiaceae & 1 \\
\hline 9. & Asclepiadaceae & 5 \\
\hline 10. & Asteraceae & 1 \\
\hline 11. & Begoniaceae & 1 \\
\hline 12. & Boraginaceae & 1 \\
\hline 13. & Cactaceae & 1 \\
\hline 14. & Caesalpiniaceae & 2 \\
\hline 15. & Cannabaceae & 1 \\
\hline 16. & Capparidaceae & 6 \\
\hline 17. & Combretaceae & 2 \\
\hline 18. & Convolvulaceae & 2 \\
\hline 19. & Cucurbitaceae & 1 \\
\hline 20. & Cyperaceae & 1 \\
\hline 21. & Dioscoreaceae & 2 \\
\hline 22. & Ebenaceae & 1 \\
\hline 23. & Euphorbiaceae & 4 \\
\hline 24. & Fabaceae & 8 \\
\hline 25. & Lamiaceae & 1 \\
\hline 26. & Malvaceae & 1 \\
\hline 27. & Meliaceae & 2 \\
\hline 28. & Menispermaceae & 2 \\
\hline 29. & Mimosaceae & 5 \\
\hline 30. & Moraceae & 8 \\
\hline 31. & Orchidaceae & 1 \\
\hline 32. & Poaceae & 4 \\
\hline 33. & Rhamnaceae & 1 \\
\hline 34. & Rubiaceae & 3 \\
\hline 35. & Rutaceae & 2 \\
\hline 36. & Salvadoraceae & 1 \\
\hline 37. & Santalaceae & 1 \\
\hline 38. & Sapindaceae & 1 \\
\hline 39. & Simaroubaceae & 1 \\
\hline 40. & Sterculiaceae & 1 \\
\hline 41. & Verbenaceae & 2 \\
\hline 42. & Vitaceae & 1 \\
\hline
\end{tabular}

\section{RESULTS}

Altogether 89 medicinally important plants belonging to 42 families and 71 genera were documented from the study area (Figs. 1 and 2). The documented medicinal plants and their vernacular name, family, status, active principle, and ethnomedicinal uses along with mode of preparation have been summarized in Table 1 and Plate 1. These plant species are used for the treatment of many diseases by tribal people. Among the 89 species of angiosperms, 80 species belong to dicotyledons and 9 species belong to monocotyledons. Dicotyledons (90\%) are dominant than the monocotyledons (10\%). Of 42 families, 37 families belong to dicotyledons and 5 families belong to monocotyledons are recorded in Table 2. Fabaceae and Moraceae were dominated with eight species.

As per the Red Data List formulated by the International Union for Conservation of Nature, around nine plants were enumerated in the Red Data List. Among these, eight plants are least con

cern and they possess valuable medicinal properties and one plant is vulnerable (Table 3).

The result of habit wise analysis shows that the tree diversity dominates in the study area. Tree recorded 31 species (35\%), shrub 24 species (27\%), herb 18 species (22\%), climber 13 species (15\%), and epiphyte 1 species (1\%) shown in Fig. 3. 
The result of part wise plant species used to cure different ailments was recorded. The plant parts such as leaves (35\%), root (16\%), whole plant $(13 \%)$, bark $(11 \%)$, stem $(9 \%)$, fruits $(8 \%)$, seeds $(6 \%)$, and

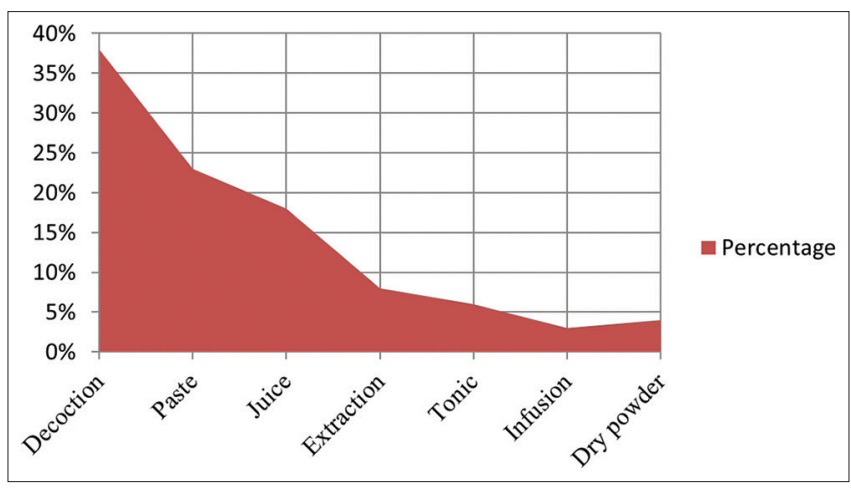

Fig. 5: Pie diagram showing the mode of the action of ethnomedicinal plants flower (2\%) were used for illness. Among these plant parts used, leaves are top in list (Fig. 4)

After the part wise analysis, the mode of the action of ethnomedicinal plants used for curing diseases in the form of decoction (38\%), paste (23\%), juice (18\%), extraction (8\%), tonic (6\%), infusion (4\%), and dry powder (3\%) shown in Fig. 5.

\section{DISCUSSION}

From this survey, we have recorded 89 plants belonging to 42 families, most of the plants belong to Fabaceae family in earlier research also supported that even they could find most of the plants belong to Fabaceae family in different regions $[11,12]$. Fabaceae is of great ethnobotanical importance in indigenous and urban communities throughout the world. Their medicinal value lies partly in their effectiveness in the treatment of a wide variety of human ailments. The variety of chemically active constituents, such as tannins, flavonoids, alkaloids, and terpenoids often found in members of this family, are substances with a high level of biological activity, and the fact that they are used extensively would suggest a pattern of global ethnomedicinal knowledge [13].

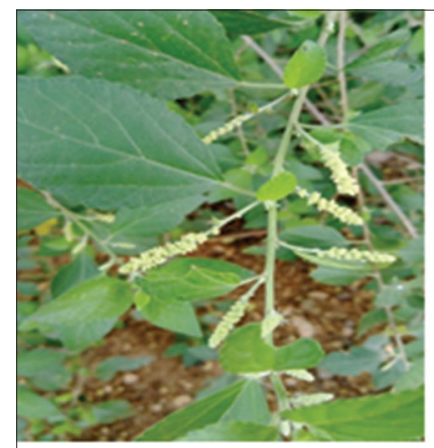

Acalypha fruticosa Forsk.

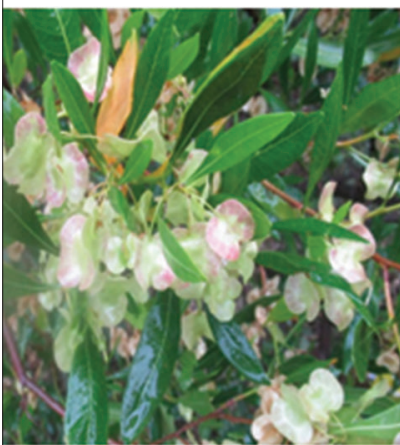

Dodonaea viscosa $\mathrm{L}$.

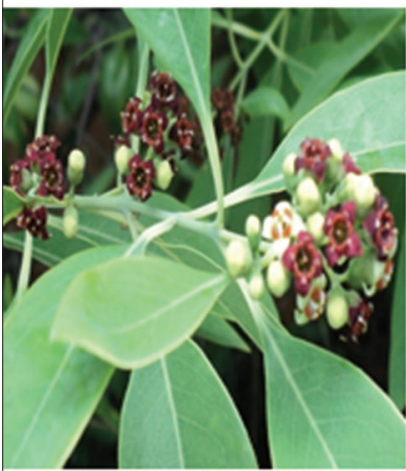

Santalum album $\mathrm{L}$.

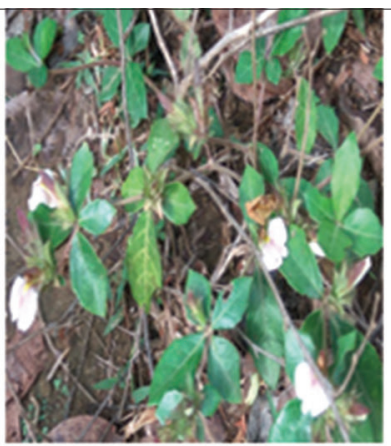

Blepharis boerhacviaefolia Pers.

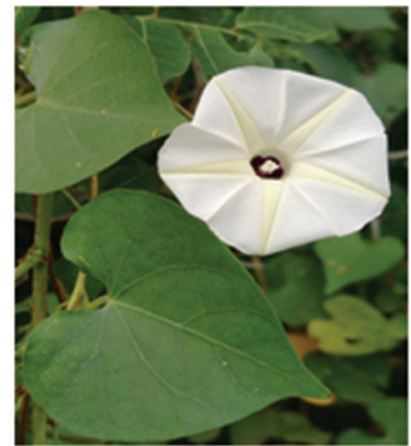

Ipomaea obscura K-Gawl.

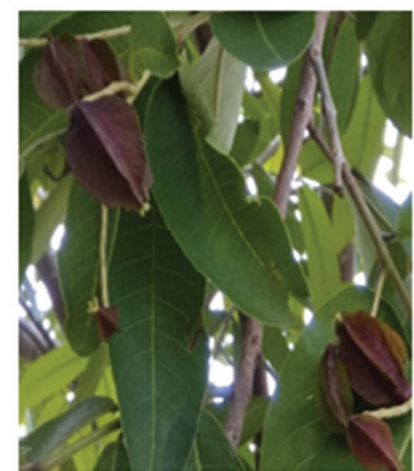

Terminalia arjuna(Roxb.)Wight \&Arn. Zizyphus oenoplia Mill.
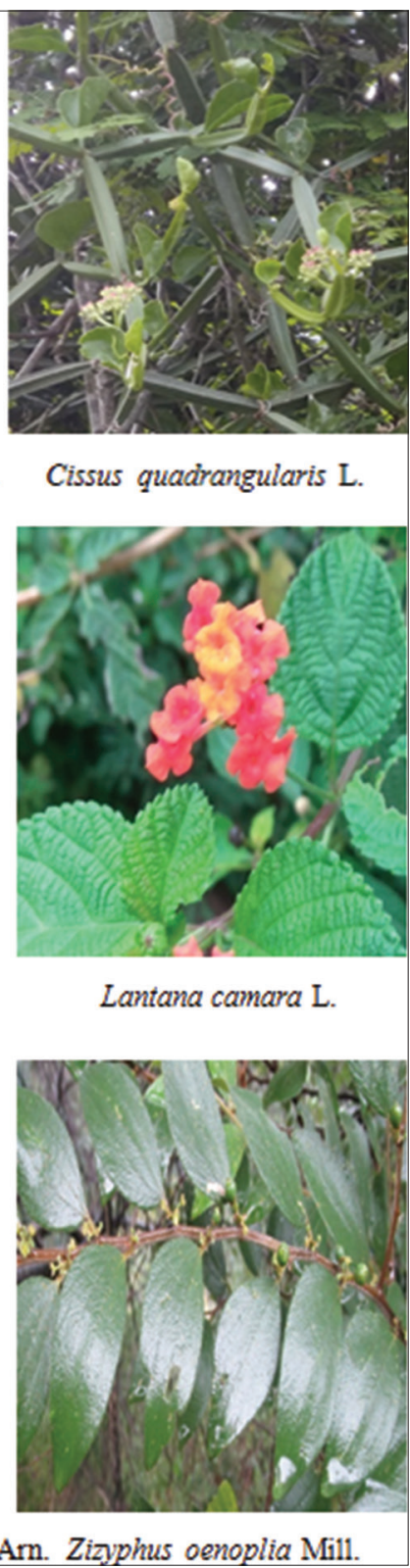

Cissus quadrangularis $\mathrm{L}$.

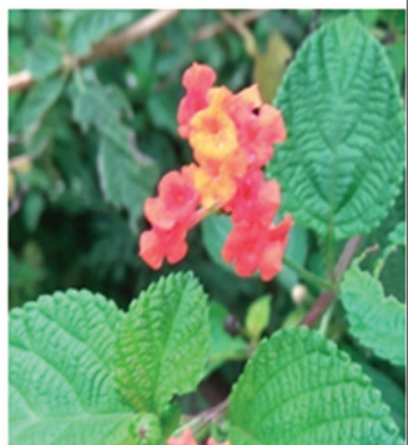

Lantana camara L.

Plate 1: Photograph of some of the surveyed ethnomedicinal plants 
Table 3: International Union for Conservation of Nature plant list category recorded in the study area

\begin{tabular}{lll}
\hline S. No. & Name of the plants & $\begin{array}{l}\text { International Union for } \\
\text { Conservation of Nature category }\end{array}$ \\
\hline 1. & Acanthus ilicifolius & LC \\
2. & Azadirachta indica & LC \\
3. & Cenchrus ciliaris & LC \\
4. & Centella asiatica & LC \\
5. & Dalbergia lanceolaria & LC \\
6. & Kyllinga triceps & LC \\
7. & Neptunia oleracea & LC \\
8. & Pongamia pinnata & LC \\
9. & Santalum album & V \\
\hline
\end{tabular}

LC: Least concern, V: Vulnerable

Leaves and roots are generally forming the most frequently used plant parts in traditional medicine $[14,15]$. Among the plant parts, the leaves are most frequently used for the treatment of diseases. This is in consonance with the findings [16]. The roots, fruits, bark, gum and latex, stem, seeds, and flowers are also used as per their availability and curing ability. Many indigenous communities throughout the world also utilized mostly leaves for the preparation of herbal medicine [17,18]. Leaves of Azadirachta indica are used for the treatment of skin diseases. The present finding is agreed with the previous report $[19,20]$. Among the plant part, the root of Aerva lanata is used for the treatment of asthma [21].

The present population is switching back to natural medicine, and in this aspect, documentation of medicinal plants is an important one. This type of documentation will help in the conservation of medicinal plants.

\section{CONCLUSION}

The present study revealed that the traditional healers of Manar beat, Karamadai range, are rich in ethnobotanical knowledge. Documentation of 89 medicinal plant species which Fabaceae and Moraceae was occurred in highest proportion of medicinal plants. From this listed plants, nine plants were enumerated in the Red Data List. Medicinal plants used in local health-care traditions are regularly becoming extinct due to overutilization, population explosion, and for other anthropogenic reasons. Therefore, it is essential to conserve such knowledge secreted in the different parts of the country and people should be promoted and protect the medicinal plants for future.

\section{ACKNOWLEDGMENTS}

The authors of this paper are gratefully acknowledged the Department of Biotechnology, New Delhi, for funding as micro grant research project. Corresponding author thanks to Tamil Nadu Collegiate Education, Chennai for providing scholarship to carry out the research work. Authors thankful to the tribal people of Manar beat, Karamadai range, Coimbatore district for sharing their valuable knowledge and help during my field work.

\section{AUTHORS' CONTRIBUTIONS}

Author 1 and 3 to investigate and supervised the findings of this work. Author 1 and 2 performed to separate the tables, figures in category wise and Author 1 performed to writing of the manuscript.

\section{CONFLICTS OF INTEREST}

There are no conflicts of interest.

\section{REFERENCES}

1. Pant S, Samant SS, Arya SC. Diversity and indigenous household remedies of the inhabitants surrounding mornaula reserve forest in West Himalaya. Indian J Tradit Knowl 2009;8:606-10.

2. Kala CP, Sajwan BS. Sustainable development of medicinal plant resources in India. ENVIS For Bull 2007;7:1-14.

3. De Luca V, Salim V, Atsumi SM, Yu F. Mining the biodiversity of plants: A revolution in the making. Science 2012;336:1658-61.

4. Juden LK. Spiritual link is part of traditional knowledge. Nature 2003;421:313.

5. Azaizeh H, Fulder S, Khalil K, Said O. Ethanomedicinal knowledge of local Arab practitioners in the middle East region. Fitoterapia 2003;74:98-108.

6. Muthu C, Ayyanar M, Raja N, Ignacimuthu S. Medicinal plants used by traditional healers in Kancheepuram district of Tamil Nadu, Indian J Ethnobiol Ethnomed 2006;2:43.

7. Gilani AH, Rahman A. Trends in ethnopharmacology. J Ethnopharmacol 2005;100:43-9.

8. Fyson PF. The Flora of the Nilgiri and Pulney Hill Tops. Vol. 3. Madras: Superintendent, Government Press; 1915-20.

9. Gamble JS, Fischer CE. Flora of the Presidency of Madras. Vol. 1-3. Calcutta; Botanical Survey of India: 1967.

10. Matthew KM. The Flora of the Tamil Nadu Carnatic. Vol. 3. Tiruchirappalli: The Rapinet Herbarium, St. Joseph's College: 1983. p. 278-9.

11. Rao SS. Ethno botanical study of medicinal plants of Sri Pancha Narasimha Swamy and Sri Matsyagiri. J Medic Plants Stud 2015;3:37-42.

12. Sadale AN, Karadge BA. Survey on ethno-medicinal plants of Ajara Tahsil, district Kolhapur, Maharashtra-(India). Trends Life Sci 2013;2:21-5.

13. Molares S, Ladio A. The Usefulness of edible and medicinal fabaceae in argentine and chilean patagonia: environmental availability and other sources of supply. Evid Based Complement Altern Med 2011;2012:1-12.

14. Rahmatullah M, Ferdausi D, Mollik AH, Jahan R, Chowdhury MH, Haque WM, et al. A survey of medicinal plants used by Kavirajes of Chalna area, Khulna district, Bangladesh. Afr J Tradit Complement Altern Med 2009;7:91-7.

15. Giday M, Asfaw Z, Elmqvist T, Woldu Z. An ethnobotanical study of medicinal plants used by the Zay People in Ethiopia. J Ethnopharmacol 2003;85:43-52.

16. Premamalini P, Sharmila S. Ethnomedicinal perspectives of botanicals used by the people of pavalamalai, gobi, Erode District, Tamil Nadu for curing various ailments. Int J Adv Herb Sci Technol 2017;3:67-75.

17. Ganesan S, Suresh N, Kesavan I. Ethnobotanical survey of Lower Palni Hills of Tamil Nadu. Indian J Tradit Knowl 2004;3:299-304.

18. Gonzalez JA, Garcia-Barriuso M, Amich F. Ethnobotanical study of medicinal plants traditionally used in the Arribes del Duero, Western Spain. J Ethnopharmacol 2010;131:343-55.

19. Suresh K, Goyal S. and Parveen F. Ethno medico botany of household remedies of Kolayat tehsil in Bikaner district, Rajasthan. Indian J Tradit Knowl 2003;2:357-65

20. Dhivya SM, Kalaichelvi K. Ethnomedicinal plants used to treat skin disease and poisonous bites by the tribals of Karamadai range, Western Ghats, Tamil Nadu, India. Int J Plant Anim Environ Sci 2016;6:53-8.

21. Sharmila S, Mownika S, Ramya EK. Survey of medicinal plants in Vellalar college for women campus, Erode, Tamil Nadu, India. Int J Pharm Sci Rev Res 2018;53:4-13. 